\title{
The recent escalation in strength of pyrethroid resistance in Anopheles coluzzi in West Africa is linked to increased expression of multiple gene families
}

Kobié H Toé ${ }^{1,2}$, Sagnon N'Falé2, Roch K Dabiré3, Hilary Ranson ${ }^{1 *}$ and Christopher M Jones ${ }^{1}$

\begin{abstract}
Background: Since 2011, the level of pyrethroid resistance in the major malaria mosquito, Anopheles coluzzi, has increased to such an extent in Burkina Faso that none of the long lasting insecticide treated nets (LLINS) currently in use throughout the country kill the local mosquito vectors. We investigated whether this observed increase was associated with transcriptional changes in field-caught Anopheles coluzzi using two independent whole-genome microarray studies, performed in 2011 and 2012.
\end{abstract}

Results: Mosquitoes were collected from south-west Burkina Faso in 2011 and 2012 and insecticide exposed or non-exposed insects were compared to laboratory susceptible colonies using whole-genome microarrays. Using a stringent filtering process we identified 136 genes, including the well-studied detoxification enzymes (p450 monoxygenases and esterases) and non-detoxification genes (e.g. cell transporters and cuticular components), associated with pyrethroid resistance, whose basal expression level increased during the timeframe of the study. A subset of these were validated by qPCR using samples from two study sites, collected over 3 years and marked increases in expression were observed each year. We hypothesise that these genes are contributing to this rapidly increasing resistance phenotype in An. coluzzi. A comprehensive analysis of the knockdown resistance (kdr) mutations (L1014S, L1014F and N1575Y) revealed that the majority of the resistance phenotype is not explained by target-site modifications.

Conclusions: Our data indicate that the recent and rapid increase in pyrethroid resistance observed in south-west Burkina Faso is associated with gene expression profiles described here. Over a third of these candidates are also overexpressed in multiple pyrethroid resistant populations of An. coluzzi from neighbouring Côte d'Ivoire. This suite of molecular markers can be used to track the spread of the extreme pyrethroid resistance phenotype that is sweeping through West Africa and to determine the functional basis of this trait.

Keywords: Anopheles coluzzi, Pyrethroid resistance, Detoxification enzymes, Transcriptomics, Vector control

\footnotetext{
* Correspondence: Hilary.Ranson@lstmed.ac.uk

'Department of Vector Biology, Liverpool School of Tropical Medicine, Liverpool L3 5QA, UK

Full list of author information is available at the end of the article
} 


\section{Background}

Resistance to the pyrethroid insecticides in Anopheles malaria mosquito vectors is now widespread throughout Sub-Saharan Africa [1]. The recent gains in reducing the burden of malaria, achieved largely by the scale-up of long lasting insecticide treated nets (LLINs), are under threat as pyrethroids represent the only insecticide class approved for use on LLINs.

The south-west of Burkina Faso was one of the first regions to report pyrethroid resistance in the local malaria vector population [2]. Since the late 1990s the level of resistance has gradually increased with the intensive agricultural activity in the area a likely contributing factor $[3,4]$. A recent survey of resistance in the village of Vallée du Kou in the south-west of the country, conducted between 2011 and 2013, has highlighted the scale of the problem with resistance levels of over 1000-fold described [5]. Laboratory assays found that none of the LLINs currently used throughout the country gave acceptable levels of mortality against local mosquitoes, raising serious concerns over the efficacy of current vector control strategies in the country.

The main resistance mechanisms to pyrethroids include target site mutations in the voltage sodium channel (the knockdown resistance mutations ( $k d r)$, L1014F, L1014S and $N 1575 Y$ ) and metabolic resistance including the overexpression of the three major detoxification enzyme families (p450 monooxygenases (p450s), glutathione-Stransferases (GSTs) and the carboxylesterases) (reviewed in [6]). While the rise and spread of the $k d r$ alleles in Anopheles gambiae throughout Sub-Saharan African are undoubtedly associated with DDT and pyrethroid resistance, they do not account for all of the variation in the phenotype $[7,8]$.

The design of microarray-based platforms for characterising gene expression profiles in Anopheles gambiae, initially based on detoxification enzymes [9], and more recently, using whole-genome wide arrays [10], has provided evidence for specific genes contributing to insecticide resistance in wild-caught An. gambiae (e.g. [11-13]). Commonly over-expressed genes have been identified in independent studies across Sub-Saharan Africa including, for example, specific P450 enzymes (e.g. CYP6M2 and CYP6P3) which have been shown to metabolize pyrethroids and other insecticide classes [10,12,14-16]. Most of these transcription studies conducted to date have compared resistant mosquitoes, collected at a single time point, to either sympatric non-exposed insects or laboratory susceptible strains. Few studies have followed transcriptional changes in wild-caught An. gambiae, from a single origin, displaying increasingly high levels of resistance at the whole-genome level.

We followed the rapid rise of deltamethrin resistance in the village of Vallée du Kou 7 (VK7) and collected mosquitoes either exposed or non-exposed to deltamethrin in 2011, 2012 and 2013 for transcriptional analysis against laboratory susceptible strains. Using a filtering process based on the over-expression of candidates in VK7, as well as an increase in expression from 2011 to 2012, we generated a list of candidate genes, including well-studied detoxification enzymes, transporters and cuticular genes that are likely contributing to the exceptionally strong pyrethroid resistance phenotype in this population. The investigation also highlighted that target site resistance, whilst highly prevalent, was not strongly associated with survival after pyrethroid exposure.

\section{Results}

\section{Deltamethrin resistance associated gene expression}

Two whole-transcriptome microarray experiments were performed on VK7 An. coluzzi collected as part of June 2011 and July 2012 bioassays (described in [5]) to identify candidate genes associated with deltamethrin resistance. This included mosquitoes that had survived more than 10 hours exposure to the diagnostic dose of pyrethroids, an unprecedented high level of resistance for malaria vectors. A detailed experimental design is given in Figure 1. In 2012, we expanded the study to include a second susceptible strain and an additional field strain (Tengrela; TEN) with a less intense resistance phenotype $(\sim 50 \%$ mortality to deltamethrin in a one-hour WHO diagnostic dose test) to improve confidence in our candidate gene list. To identify candidate genes in VK7 we made three underlying assumptions: a) candidate resistance genes are more highly expressed in resistant field populations versus susceptible lab colonies (MAL originating from Mali \& NG originating from Cameroon), b) the same underlying mechanisms were responsible for resistance in 2011 and 2012 and c) genes conferring pyrethroid resistance would be more highly over-expressed in VK7 compared to TEN. A detailed analysis schema is provided in Figure 2 and the Methods.

\section{Genes over-expressed in resistant field populations}

The filtering approach which compared all field resistant populations to the susceptible laboratory strains, using data from both 2011 and 2012, left 605 probes (representing 487 genes) (Additional file 1) including 15 cytochrome P450s, 9 glutathione S-transferases (GSTs), 3 carboxylesterases and many other non-detoxification genes. The top five over-expressed detoxification genes based on the VK7/MAL comparison in 2012 included GSTE2 (AGAP009194, four probes, average $\mathrm{FC}=9.48$ ), CYP4G16 (AGAP001076, four transcripts, average $\mathrm{FC}=$ 6.30), GSTS1_1 (AGAP010404, three probes, average $\mathrm{FC}=3.7$ ), CYP9J5 (AGAP012296, $\mathrm{FC}=3.03$ ) and CYP6P1 (AGAP002868, four probes, average $\mathrm{FC}=2.69$ ). The most over-expressed non-detoxification genes consisted of an 


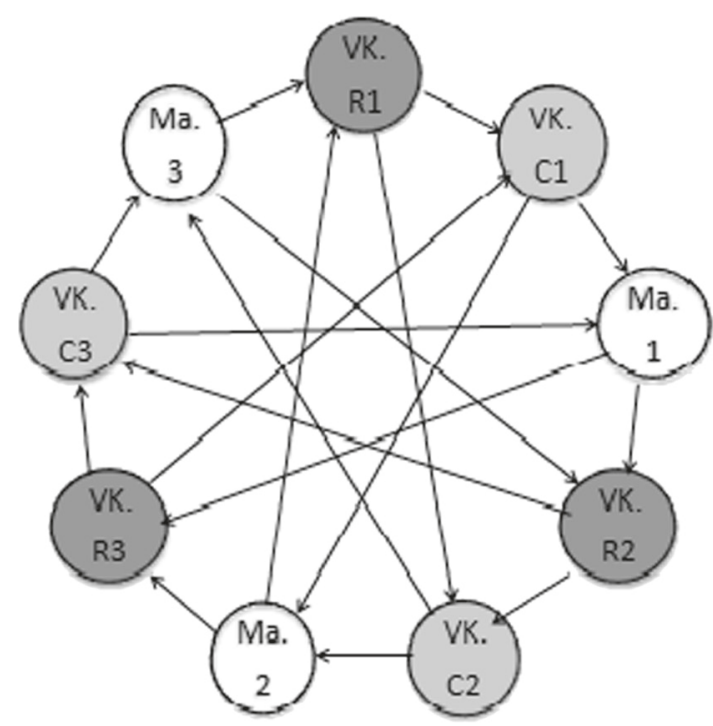

2011

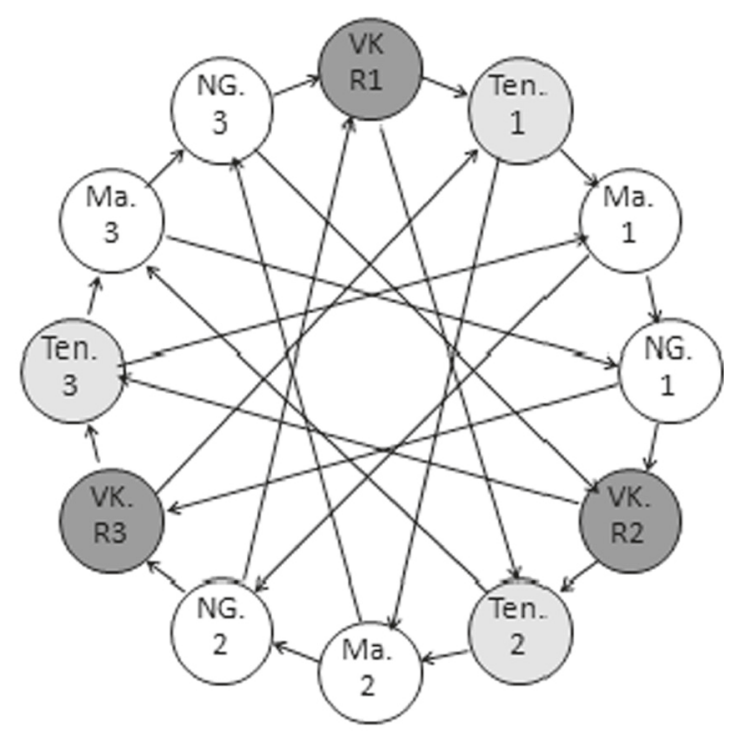

2012

Figure 1 Interwoven loop designs for microarray experiments performed in 2011 and 2012. In 2011, deltamethrin selected mosquitoes from VK7 (VKR; $L T_{50}=254$ min), unexposed mosquitoes from VK7 (VKC) and the Mali susceptible lab strain (MAL) were compared. In 2012, four mosquitoes populations were compared including deltamethrin selected mosquitoes from VK7 (VKR; 600 mins exposure), unexposed mosquitoes from a deltamethrin resistant population approximately $120 \mathrm{~km}$ from VK7, Tengrela (TEN), the susceptible MAL strain and a second fully susceptible lab strain from N'Gousso, Cameroon (NG). The direction of the arrows represents a cy3 to cy5 hybridisation and three biological replicates were used for each comparison.

ATP synthase (AGAP006879, FC = 22.30), glycoside hydrolase (AGAP009110, $F C=9.51$ ), a cuticular protein with chitin binding domains CPAP3-A1b (AGAP000987, $\mathrm{FC}=9.35)$ and a takeout protein associated with insect circadian clocks (AGAP004262, FC =11.48). Additional over-expressed genes, which were also up-regulated in later filtering steps (see below), included an aquaporin (AGAP010326, FC = 7.97), chymotrypsin-1 (AGAP006709, $\mathrm{FC}=5.18$ ) and the carboxylesterase, COEAE3G (AGAP 006724-RA, $\mathrm{FC}=2.56$, four probes) (Additional file 1 ).

\section{Genes expressed at higher levels in deltamethrin survivors from 2012 compared to 2011}

Out of the 605 probes over-expressed in all the field resistant populations compared to the laboratory susceptible strains, 374 probes had a higher fold change in 2012 than in 2011 based on the VK7/MAL comparison (Step E in Figure 2; Additional file 2). As resistance levels increased dramatically between these years we hypothesised that this probe list may include genes contributing to the resistance phenotype. The five GSTs genes (GSTE2 (AGAP009194, FC = 9.48), GSTMS3 (AGAP009946, FC = 2.52), GSTS1_2 (AF513639, FC = 2.42), GSTM1 (AGAP000165, FC = 2.24), and GSTE5 (AGAP009192, FC = 2.37)), five out the fifteen cytochrome P450s (CYP4G16, CYP6P1, CYP9J5, CYP6Z3 (AGAP008217, FC = 2.36) and CYP9M1 (AGAP009363,
$\mathrm{FC}=2.27)$ ) and two of out the three carboxylesterases (COEAE3G (AGAP006724, FC = 2.41) and COEAE4G (AGAP006725, FC = 1.37)) remained after this filtering approach. The highest fold-change differences between 2012 and 2011 were observed for genes already showing high constitutive expression including the chymotrypsin1 (AGAP006709, FC = 3.64), aquaporin (AGAP010326, 2.60), cuticular protein $C P A P 3-A 1 b$ (AGAP000987, $\mathrm{FC}=$ 2.19) and the ATP synthase (AGAP006879, FC difference $=2.14$ ). The P450, CYP4G16 (AGAP001076), which has been implicated in pyrethroid resistance elsewhere [17], also showed an increase in expression in 2012.

Seven of the nine candidate genes chosen for qPCR validation were expressed at higher levels in VK7 in 2012 compared to 2011 (two tailed $t$-test, $P<0.05$ ) (Figure 3 ).

\section{Genes expressed at higher levels in VK7 compared to TEN} We included $A n$. coluzzi mosquitoes from Tengrela (TEN) in the 2012 experiment to see whether a common set of genes were up-regulated in pyrethroid resistant mosquitoes from VK7 and TEN. Considering that resistance is higher in VK7 than TEN, we only retained probes significantly over-expressed in VK7 compared to TEN. This gave a final candidate gene list containing 157 probes (Additional file 3). A hierarchical clustering analysis based on the expression profiles of three comparisons between VK7/MAL (2011), VK7/MAL (2012) and VK7/NG (2012) 


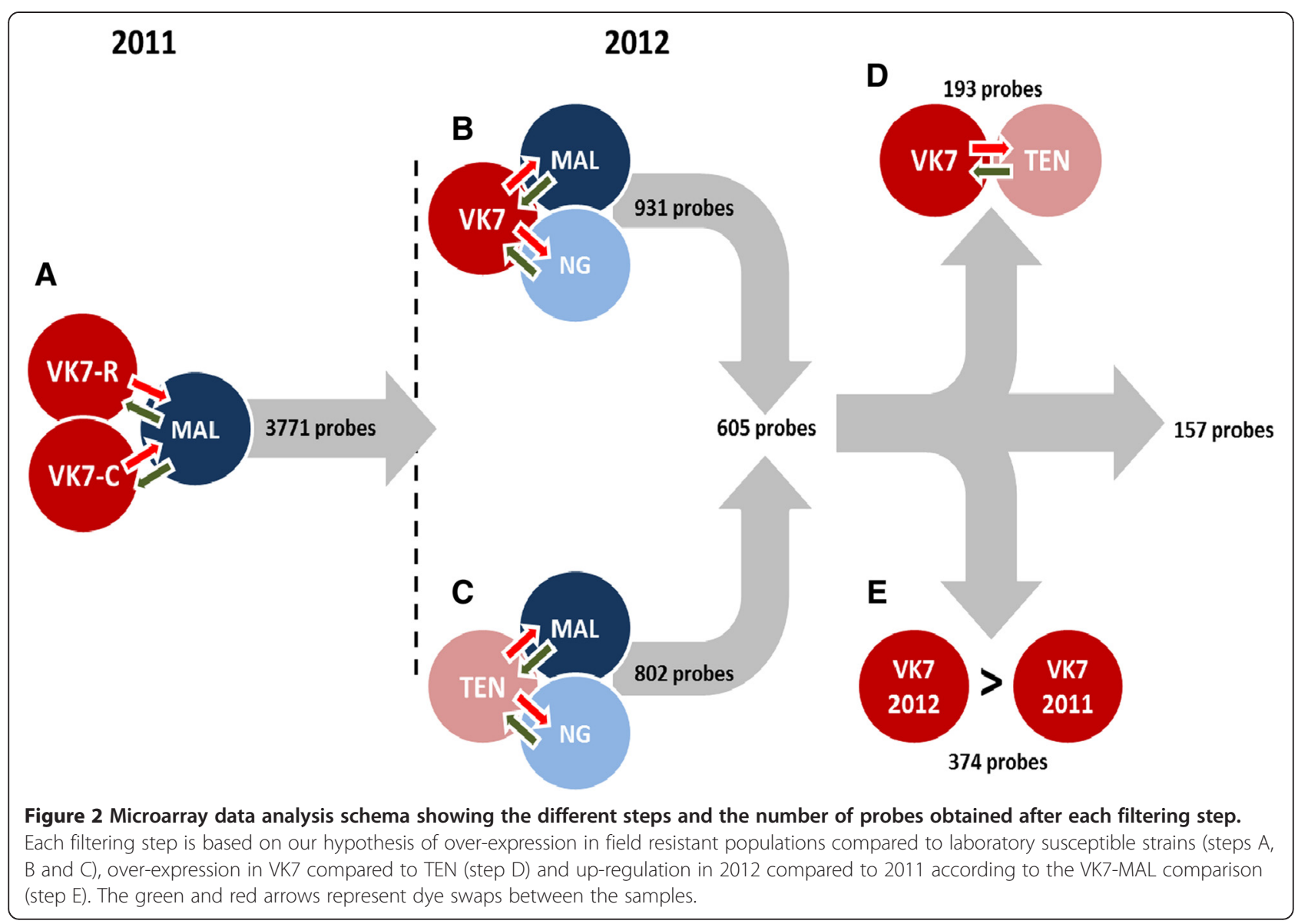

is presented in Figure 4. If putative alternative transcripts of the same gene and duplicate probes are removed, the candidate gene list reduces further to 136 unique genes. Several of the detoxification genes having higher FC in 2012, including CYP4G16, CYP9J5, CYP9M1, COEAE3G, GSTE5, were retained in this list as well the components of the cuticle (e.g. CPR 73, CPAPA3-A1a and CPAPA3$A 1 b)$ and the chymotrypsin-1, aquaporin and ATP synthase (Additional file 3).

Genes expressed at higher levels in TEN compared to VK7 Interestingly, several detoxification genes were more highly expressed in TEN than VK7 in 2012 (and thus were filtered out of our final candidate list for VK7) (Additional file 4). Of all genes up-regulated in TEN $(\mathrm{N}=171)$, the highest fold changes were for CYP6Z3 (AGAP008217, $\mathrm{FC}=2.55$, four probes) and CYP6P4 (AGAP002867, FC = 2.32). Both of these p450s also showed a significant increase in basal expression in VK7 from 2012 to 2013 (Figure 3) suggesting that, given their reported role in pyrethroid resistance $[15,16]$, these enzymes may be involved in resistance in both Tengrela and VK7.

\section{Target-site resistance}

All three $k d r$ mutations (L1014F, L1014S and N1575Y) in the voltage gated sodium channel have been reported in An. gambiae s.l from south-west Burkina Faso [3,19]. Dead and surviving VK7 mosquitoes after exposure to the $\mathrm{LT}_{50}$ of deltamethrin were screened for each mutation to determine whether, in addition to the increases in gene expression described above, target-site resistance contributes to the strong resistance phenotype. The $1014 S k d r$ allele was not found in any sample. The frequency of the $1014 \mathrm{~F}$ allele in the 2011-2012 control samples of $A n$. coluzzi was high (0.823 to 0.880$)$ but did not differ significantly between date of collection (Fisher's exact test, two tailed $P$ value $>0.05$ ) (Table 1 ). A similar frequency of 0.877 of $1014 F$ was observed in 2012 from Tengrela (Table 1). Samples collected in July 2013 from the different breeding sites in VK7 and Tengrela showed a similar frequency (chi-square test $=1.69, P=0.43$ ) (Table 1). The $1575 Y k d r$ allele had a frequency of between 0.237 and 0.355 during the collection period in both VK7 and Tengrela. No significant increase or decrease in the frequency of $N 1575 Y$ was observed during this time (Table 1). 


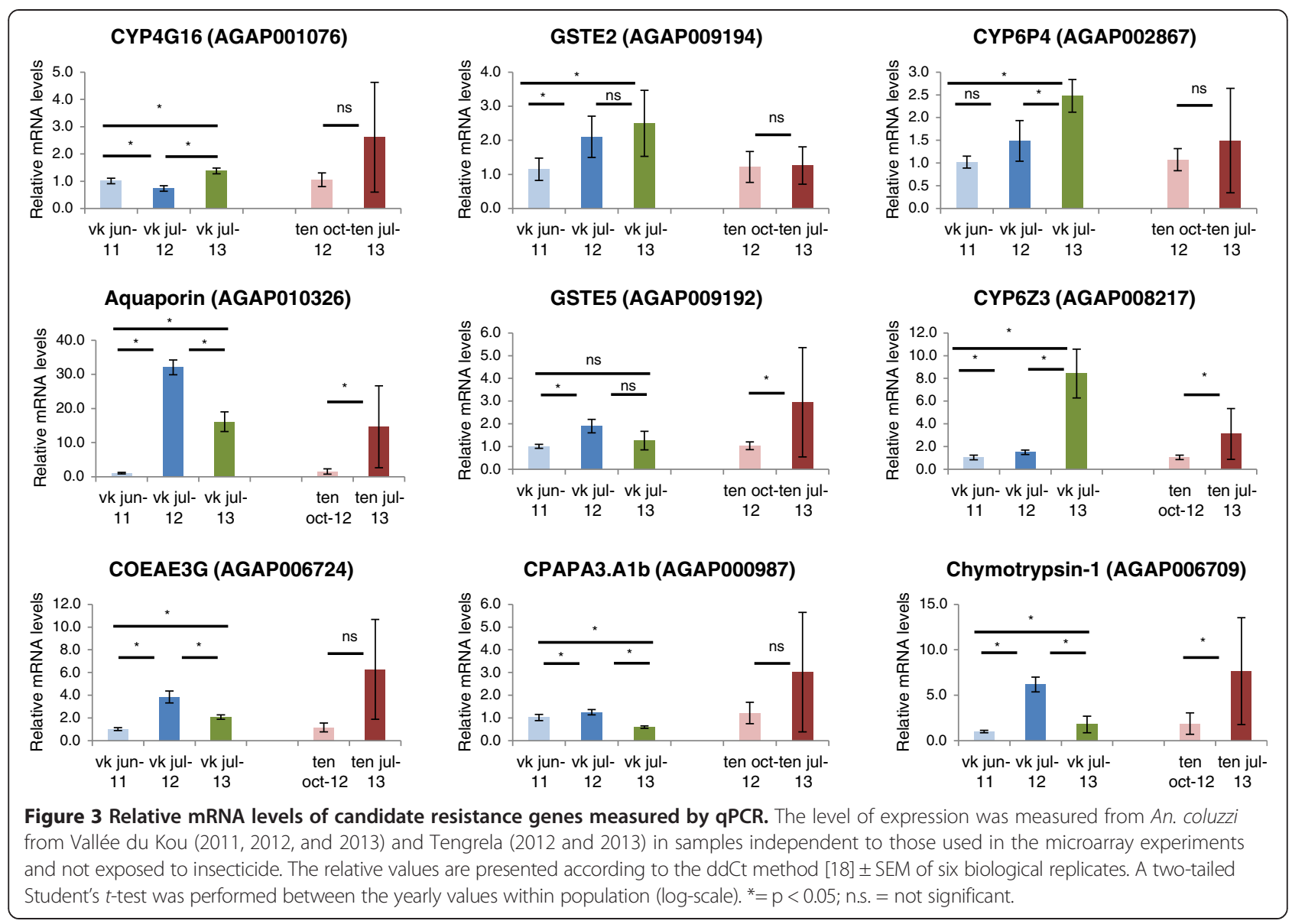

Deltamethrin resistance and $k d r$ haplotype association

In almost all cases the frequencies of $1014 \mathrm{~F}$ and $1575 \mathrm{Y}$ were higher in mosquitoes surviving insecticide exposure but the presence of the $1014 \mathrm{~F}$ allele was only significantly associated with deltamethrin survival in one round (October 2011) $(P=0.04)$ and in the case of $1575 Y$, an association with deltamethrin survival was observed in two rounds of collection $(P=0.015$ and 0.043 in October 2011 and June 2012 respectively (Table 1)). $1575 Y$ occurs exclusively on a haplotypic background of $1014 \mathrm{~F}$ and a stronger association of the haplotype, rather than single alleles, has been demonstrated for DDT and pyrethroid resistance in some cases [19]. Haplotypic association tests revealed that the presence of both $1014 \mathrm{~F}$ and $1575 Y$ alleles increased the OR of surviving the deltamethrin $\mathrm{LT}_{50}$ in VK7 from all three collection rounds but the difference was only significant for samples collected in October 2011 and in June 2012 (OR $2.68(P=0.007)$ and $3.00(P=0.046)$ respectively (Figure 5$))$.

\section{Discussion}

In this study, we applied a robust microarray-approach to determine the transcriptional profile of an An. coluzzi population undergoing further selection on top of an already strong pyrethroid resistance phenotype. We previously documented that deltamethrin resistance ratios (the $\mathrm{LT}_{50}$ of the local resistant population divided by the $\mathrm{LT}_{50}$ susceptible strain) increased from 54 to 730 in a single calendar year from the village of VK7 in Burkina Faso [5]. By analysing transcription levels of An. coluzzi from VK7 in both 2011 and 2012, this provided a unique opportunity to determine whether gene expression levels were simultaneously rising with pyrethroid resistance in a single wild-caught population of $A n$. coluzzi from south-west Burkina Faso.

\section{Candidate genes for deltamethrin resistance in VK7}

According to our hypotheses of over-expression in field resistant mosquitoes compared to laboratory susceptible strains, increased expression in VK7 from 2011 to 2012, and finally, higher expression in VK7 than the more moderate resistance phenotype displayed in nearby Tengrela, we filtered microarray probes down to a final list of 157 representing 136 genes which are potentially contributing to deltamethrin resistance in VK7. These encode enzymes and proteins with both detoxification and non-detoxification functions. This gene list was compared to data generated using the same microarray 


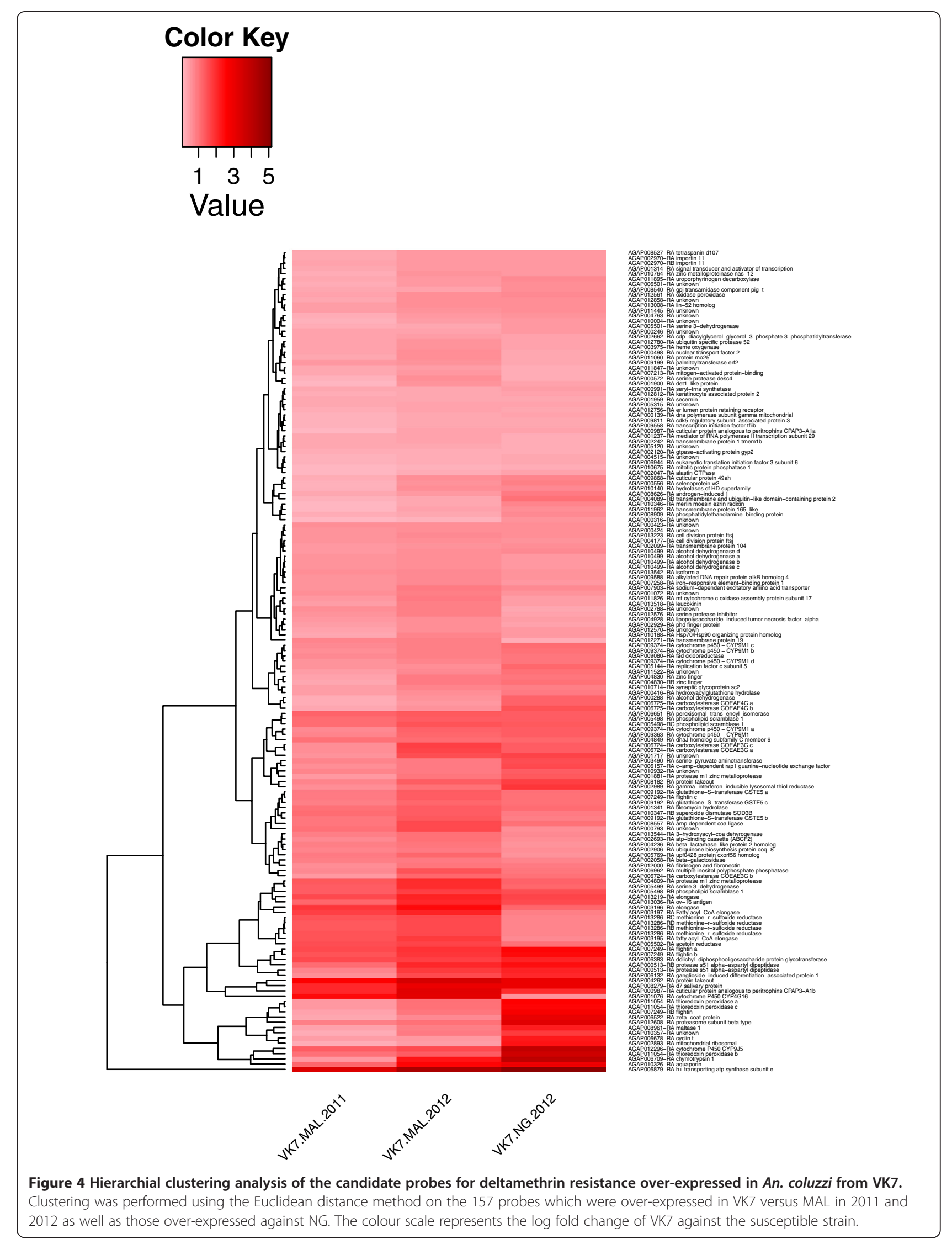


Table 1 The frequency of the L1014F and N1575Y mutations in An. coluzzii tested in deltamethrin resistance bioassays

\begin{tabular}{|c|c|c|c|c|c|c|c|c|c|c|c|}
\hline Period & Status & $\mathrm{L}$ & $F$ & n (alleles) & (f) L1014F & $95 \% \mathrm{Cl}$ & $\mathrm{N}$ & $\mathrm{Y}$ & n (alleles) & (f) $\mathrm{N} 1575 \mathrm{Y}$ & $95 \% \mathrm{Cl}$ \\
\hline \multicolumn{12}{|l|}{ VK7 } \\
\hline \multirow[t]{3}{*}{ Jul-11 } & Control & 22 & 102 & 124 & 0.823 & $0.744-0.886$ & 80 & 44 & 124 & 0.355 & $0.276-0.442$ \\
\hline & Dead & 23 & 97 & 120 & 0.808 & $0.728-0.870$ & 92 & 34 & 126 & 0.270 & $0.200-0.354$ \\
\hline & Survivors & 19 & 95 & 114 & 0.833 & $0.754-0.892$ & 80 & 30 & 110 & 0.273 & $0.198-0.363$ \\
\hline \multirow[t]{3}{*}{ Oct-11 } & Control & 27 & 163 & 190 & 0.858 & $0.801-0.901$ & 134 & 56 & 190 & 0.295 & $0.234-0.363$ \\
\hline & Dead & 42 & 194 & 236 & 0.822 & $0.768-0.866$ & 174 & 60 & 234 & 0.256 & $0.205-0.316$ \\
\hline & Survivors & 15 & 133 & 148 & 0.899* & $0.839-0.939$ & 91 & 55 & 146 & $0.377^{*}$ & $0.302-0.458$ \\
\hline \multirow[t]{3}{*}{ Jun-12 } & Control & 25 & 183 & 208 & 0.880 & $0.828-0.918$ & 151 & 55 & 206 & 0.267 & $0.211-0.331$ \\
\hline & Dead & 9 & 45 & 54 & 0.833 & $0.710-0.912$ & 42 & 12 & 54 & 0.222 & $0.131-0.351$ \\
\hline & Survivors & 14 & 132 & 146 & 0.904 & $0.844-0.943$ & 90 & 56 & 146 & $0.384^{*}$ & $0.309-0.465$ \\
\hline Jul-13 & Control & 16 & 126 & 142 & 0.887 & $0.823-0.934$ & 109 & 33 & 142 & 0.232 & $0.166-0.311$ \\
\hline \multicolumn{12}{|c|}{ Tengrela } \\
\hline \multirow[t]{3}{*}{ Jul-12 } & Control & 14 & 100 & 114 & 0.877 & $0.803-0.931$ & 87 & 27 & 114 & 0.237 & $0.162-0.326$ \\
\hline & Dead & 18 & 80 & 98 & 0.816 & $0.725-0.887$ & 66 & 32 & 98 & 0.327 & $0.235-0.429$ \\
\hline & Survivors & 11 & 81 & 92 & 0.880 & $0.796-0.939$ & 66 & 26 & 92 & 0.283 & $0.194-0.386$ \\
\hline Jul-13 & Control & 24 & 124 & 148 & 0.838 & $0.768-0.893$ & 109 & 39 & 148 & 0.264 & $0.194-0.342$ \\
\hline
\end{tabular}

Allele frequencies with $95 \% \mathrm{Cl}$ of $1014 \mathrm{~F}$ and $1575 \mathrm{Y} \mathrm{kdr}$ alleles in An. coluzzi from Tengrela and VK7. VK7 samples are stratified according to deltamethrin exposure (unexposed control samples and mosquitoes that died or survived exposure to an approximate $\mathrm{LT}_{50}$ ). In Tengrela the exposure time was one hour. Sample sets where allele frequency is significantly associated with insecticide survival $(P<0.05)$ are marked by *.

platform, comparing gene expression in deltamethrin survivors of An. coluzzi from three independent sites in Cote d'Ivoire, with the N'Gousso susceptible strain (C Strode, unpublished data, ArrayExpress Accession numbers E-MTAB-3210, E-MTAB-3211, E-MTAB-3212). One third of the 136 genes associated with deltamethrin resistance in Burkina Faso were also found over expressed in all three populations from Côte d'Ivoire, including CYP4G16 and the cuticular proteins CPAP3-A1a and $C P A P 3-A 1 b$ (Additional file 5 ). This suggests that similar mechanisms could underlie the pyrethroid resistance that is spreading across Western Africa.

Three P450s were present in the list of VK7 candidate probes (CYP4G16, CYP9J5, and CYP9M1). CYP4G16 is becoming increasingly associated with pyrethroid resistance with evidence for over-expression in another major vector of the An. gambiae complex, An. arabiensis $[17,20]$. Although CYP4G16 does not metabolize pyrethroids directly, it may play a role in enhancing cuticular hydrocarbon synthesis as demonstrated in members of the CYP4G family previously $[21,22]$. The presence of additional genes with putative roles in cuticular hydrocarbon synthesis (two elongases (AGAP003196-RA \& AGAP013219-RA), 3-hydroxyacyl-coa dehydrogenase (AGAP013544-RA) and fatty acyl-CoA elongase (AGAP003195-RA)) strengthens the support for the involvement of this pathway, and CYP4G16, in pyrethroid resistance in An. gambiae, although further functional validation is needed. Furthermore, the Cuticular Proteins Analogous to Peritrophins (CPAP) class of proteins are expressed in cuticule forming tissues and maintain the structural integrity of the cuticle [23]. Two members of the CPAP3 family were consistently
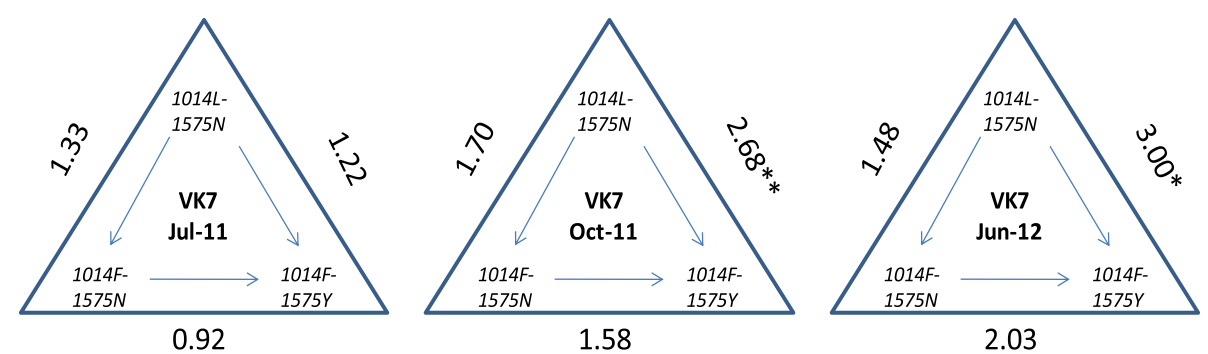

Figure 5 Haplotypic association tests for the three $k d r$ haplotypes (1014L-1575N, 1014F-1575N and 1014 F-1575Y) with survival of VK7 An. coluzzi to deltamethrin $\mathrm{LT}_{\mathbf{5 0}}$. Odds ratios $(\mathrm{OR})$ are represented with the level of significance and the arrows within the triangles show the direction of the OR calculation. ${ }^{*} \mathrm{p}<0.05,{ }^{* *} \mathrm{p}<0.01$. 
over-expressed in VK7 (CPAP3-A1a (AGAP000989-RA) and CPAP3-A1b AGAP000987-RA)). CPAP3-A1b was recently over-expressed in $A n$. gambiae from an agricultural area in Northern Tanzania [24] and therefore this protein class warrants further investigation as part of any cuticular resistance hypothesis in this species.

The aquaporin (AGAP010326-RA) and chymotrypsin1 (AGAP006709-RA) were both significantly and highly over-expressed in VK7. The precise role for these proteins in insecticide resistance remains unclear. Aquaporins are ubiquitous water transporting membrane proteins found in the Malpighian tubule system of insects [25]. The aquaporin over-transcribed in this study was also the most significantly and highly over-expressed gene in a genomewide transcriptional profile of an extremely resistant DDT strain of An. coluzzi from Ghana (survival after $6 \mathrm{hr}$ exposure to DDT 4\%) [10]. The presence of aquaporins in the Malpighian tubules, which are also a potential site for p450-mediated metabolism [26], may represent an efflux/ excretion system for insecticide detoxification. In the field of drug resistance, the loss of the activity of one of these transporters leads to resistance to melaminophenyl arsenic (MPA) and pentamidine treatment in the African Trypanosomiasis parasite (Trypanosoma brucei) [27]. Chymotrypsins are serine proteases secreted in the midgut of insects [28]. Chymotrypsin transfected Culex pipiens pallens cells treated with deltamethrin showed greater viability compared to control cells [29] but it is not clear how this enzyme class would directly or indirectly increase insecticide tolerance.

Other metabolic enzymes were present in our VK7 candidate list including two carboxylesterases (COEAE3G, COEAE4G) and a GST (GSTE5). The expression of two of these genes, COEAE3G and GSTE5, was greater in VK7 in 2012 according to our qPCR assays. Although the upregulation of these enzymes has been shown in pyrethroid and DDT resistant mosquito populations elsewhere $[12,13,30]$, it is more likely that these enzymes either play a secondary role or are involved in resistance to other compounds. For example, members of the epsilon class of GSTs, and in particular GSTE2, can metabolise DDT [31] and we included this in the qPCR assays given the strong evidence in its role in DDT resistance and the elevated expression in the field An. coluzzi from VK7 and Tengrela. The VK7 population displays intense DDT resistance with 50 hours of DDT exposure inducing only $34.3 \%$ of mortality (Toé et al, data not shown) and it is possible that GSTE2 and/or GSTE5 are involved in resistance to DDT rather than to pyrethroids [11,32].

As part of our filtering process to determine candidate genes from VK7, we decided to choose only those genes over-expressed in VK7 compared to Tengrela, however, when analysing this data we noticed that two strong candidate P450s for pyrethroid resistance, CYP6Z3 and
CYP6P4 [15,16], were more highly expressed in Tengrela in 2012. However, by 2013, a year which saw pyrethroid resistance ratios exceed 1000× in VK7 [5], the expression of these two P450s in VK7 increased significantly, suggesting that these P450s may be contributing to the resistance phenotype in both locations.

The vast majority of transcriptional studies investigating the association of gene expression with insecticide resistance have focussed on the over-expression of candidate genes. While there is a large body of evidence to support this approach, the down-regulation of genes as part of key biological pathways should not be ignored. Additional file 6 provides a list of the 291 probes (254 genes) down-regulated in all resistant strains versus the susceptible lab colonies including those under-transcribed in VK7 compared to TEN. This list includes many transporter proteins (including odorant binding proteins) and ion channels plus a small number of cytochrome P450 genes.

\section{The $1014 F-1575 Y$ kdr haplotype only accounts for a small portion of the resistance phenotype in VK7}

The link between the $k d r 1014 F$ allele in the target sodium channel and the ability to survive pyrethroids or DDT is clear with selective sweeps acting upon this allele throughout different parts of Africa [7]. However, it is also evident that $k d r$ alone does not account for all of the phenotypic variation in resistance. In this study, $1014 \mathrm{~F}$ or $1575 \mathrm{Y}$ alone were only mildly associated with an increased ability to survive the $\mathrm{LT}_{50}$ for deltamethrin. The $1014 F k d r$ diagnostic might only have a limited impact in populations with high levels of pyrethroid resistance, as reported here, and elsewhere in West Africa [33]. The N1575Y mutation is found exclusively on a $1014 F$ haplotypic background and this haplotype has been shown to confer additional selective advantage in presence of insecticides [19]. The 1014F-1575Y haplotype did provide some protection to deltamethrin (Figure 5) but the highest OR observed of 3.00 does not account for the extremely high resistance ratios observed in VK7.

\section{Conclusions}

Using a quantitative approach to resistance monitoring we recently documented the highest level of deltamethrin resistance recorded in field-caught An. gambiae to date. Transcriptional profiling of these resistant mosquitoes has identified a suite of candidate genes, which can be used to monitor resistance levels as attempts are made to mitigate against development of additional resistance in the future using new vector control tools [34]. Furthermore, the findings presented here provide evidence for additional mechanisms which might be contributing to such a strong phenotype (i.e. cuticular synthesis) and urgently need further investigation. 


\section{Methods}

\section{Mosquito collections and genotyping}

All mosquitoes used for the genotyping and microarrays were collected in June of 2011 and July of 2012 from breeding sites as larvae and reared to adults at the insectaries of CNRFP. In 2013, these included adults emerging from larvae collected in paddy fields and open puddles and reared separately. Mosquitoes were collected from two study sites of VK7 $\left(11^{\circ} 39^{\prime} \mathrm{N}, 04^{\circ} 41^{\prime} \mathrm{W}\right)$ and Tengrela $\left(10^{\circ} 40^{\prime} \mathrm{N}, 4^{\circ} 50^{\prime} \mathrm{W}\right)$ where the level of insecticide resistance has been characterised and its impact on LLIN efficacy reported [5]. Prior to RNA extraction, two or three legs of each individual mosquito taken from deltamethrin-treated or non-treated bioassays were removed for PCR identification and genotyping before placing the remaining body in RNA later (Sigma). Genomic DNA was extracted from mosquito legs by boiling in $20 \mu \mathrm{l} 1 \times$ PCR buffer or from whole mosquitoes using Livak buffer [35]. Identification of species within the An. gambiae complex was performed using SINE-PCR [36]. The frequencies of the 1014F, $1014 \mathrm{~S}$ and $1575 \mathrm{Y} k d r$ mutations were assessed using TaqMan ${ }^{\circ}$ PCR assays [19,37]. Genotyping was performed on all the control, surviving and dead mosquitoes exposed to the $\mathrm{LT}_{50}$. Allele frequencies were compared between dead and surviving insects using a $2 \times 2$ contingency table (Fisher's exact test, two-tailed $P$-value). Haplotype association tests between 1014L-1575N, 1014F-1575N and $1014 F-1575 Y$ and survival to deltamethrin were performed using the Haploview software v.4.2 [38] and the odds ratio for survival and chi-square tests for association conducted for each round of insecticide testing.

\section{Whole genome microarray study design}

All the mosquitoes used for microarrays and the qPCR assays were confirmed as An. coluzzi (formerly $A n$. gambiae M molecular form) [39]. Two independent wholegenome microarray experiments were performed to identify candidate genes associated with deltamethrin resistance in VK7 using an interwoven a loop design (Figure 1) [40]. The interwoven loop design used in our experiments has been shown to provide more power in detecting small differences in gene expression [41]. The first experiment compared VK7 mosquitoes collected in 2011 that were a) unexposed to insecticides (VKC) and b) had survived a $4 \mathrm{hr}$ exposure to $0.05 \%$ deltamethrin (VKR) with an insecticide susceptible strain from Mali (MAL). In the second experiment, VK7 mosquitoes collected in the following summer in 2012 which had survived a $10 \mathrm{hr}$ exposure to deltamethrin (0.05\%) (VKR) were compared to MAL and a second fully susceptible strain from N'Gousso (NG) (Cameroon). In this second experiment, an unexposed population from Tengrela (TEN), a rice field area located approximately $120 \mathrm{~km}$ from VK7 which showed 50\% mortality following one hour exposure to deltamethrin, was added to the design. The objective of this expanded study in 2012 was to improve the confidence in our candidate gene list by including a second susceptible strain and an additional field strain with a less intense resistance phenotype. Each biological replicate in the microarray consisted of ten non-blood females and were 5 days old.

\section{RNA extraction and microarray data analysis}

Mosquitoes exposed to insecticide were retained for a further 24 hours prior to storage in RNA later (Sigma) to minimise any induction effect from insecticide exposure and unexposed mosquitoes were exposed to untreated control papers. Total RNA was extracted from pools of ten mosquitoes using RNAqueous ${ }^{\circ}$-4PCR Kit for isolation of DNAse-free RNA (Ambion) according to the manufacturer's protocol. The quality and quantity of the RNA used for the microarrays was assessed using a Nanodrop spectrophotometer (Nanodrop Technologies) and 2100 Bioanalyser (Agilent Technologies). cRNA (100 ng) was synthesised and labelled with cyanine 5 (cy5) or cyanine 3 (cy3) using the Two-Color Low Input Quick Amp Labelling Kit (Agilent Technologies) according to the manufacturer's instructions. Labelled cRNA were then column purified (QIAGEN) and eluted in $30 \mu \mathrm{l}$ of RNase free water. Labelled cRNA samples (300 ng) were hybridized to the $8 \times 15 \mathrm{~K}$ Aligent whole-transcriptome $A n$. gambiae microarray chip (A-MEXP-2196) [10] for $17 \mathrm{~h}$ at $65^{\circ} \mathrm{C}$. Slide washing, scanning and feature extraction were performed as described previously [10].

The raw array data were normalised using LIMMA [42] prior to applying a MAANOVA model in R software [43]. The 2011 and 2012 results were combined and a hypothesis driven filtering approach of the probe sets applied to identify candidate genes in VK7. A schema of our approach is given in Figure 2. Under our hypothesis of greater expression in resistant mosquitoes, genes were considered candidates if probes were significant (false discovery rate adjusted $P$ value $(\mathrm{q})<0.05$ ) and the fold-changes were consistently up-regulated in all pair-wise comparisons between resistant versus susceptible mosquitoes (Steps A-C, Figure 2). This provided a set of baseline candidate probes from which probes were filtered further if they were significantly over-expressed $(\mathrm{q}<0.05)$ in VK7 compared to TEN (Step D, Figure 2) and showed greater expression in 2012 compared to 2011 (Step E, Figure 2).

\section{qPCR for candidate gene expression in VK7 and TEN}

The expression of a selection of nine genes from the microarray analyses was validated using reverse-transcription quantitative PCR (RT-qPCR). All mosquito samples were unexposed to insecticides. Each replicate was independent of the samples used in the microarray to provide external validation of the expression data. Our hypothesis was 
based on the rapid rise of resistance in VK7 during the time period between June 2011 and July 2013 and therefore we anticipated expression of candidates to concurrently rise. According to this hypothesis we were concerned primarily with the changes in expression levels in the resistant sites rather than pair wise comparisons with susceptible strains. As a result only samples from VK7 and Tengrela were tested in the qPCR. Primers were designed using PrimerBLAST tool (NCBI: http://www.ncbi.nlm.nih.gov/tools/ primer-blast/) against the An. gambiae PEST sequence and at least two primer sets were used to assess their efficiency and specificity by running a standard curve over a five-fold serial dilution (Additional file 7). Approximately $600 \mathrm{ng}$ of RNA was reverse transcribed to first strand cDNA using SuperScript ${ }^{\text {tw }}$ III reverse transcriptase (Invitrogen). All six biological replicates for each population were run in triplicate using $5 \mu \mathrm{l}$ of input cDNA diluted 10-fold, $2 \times$ SYBR Brilliant III (Aligent Technologies) and $300 \mathrm{nM}$ of the forward and reverse primer on the Mx3005P qPCR system (Aligent Technologies). The thermal profile for each reaction was $95^{\circ} \mathrm{C}$ for $3 \mathrm{~min}$ followed by 40 cycles of $95^{\circ} \mathrm{C}$ for $10 \mathrm{~s}$ and $60^{\circ} \mathrm{C}$ for $10 \mathrm{~s}$. The qPCR data were analysed according to the ddCt method [18] relative to the average of three housekeeping genes encoding a ubiquitin protein (AGAP007927), an elongation factor (AGAP005128) and the $\mathrm{S} 7$ ribosomal protein (AGAP010592). The qPCR efficiency of each primer set was incorporated into the reaction. The $\mathrm{Ct}$ data were log-transformed to ensure a normal distribution of the data prior to statistical analysis. To determine whether a significant changes in expression levels occurred during the timeframe of the study (in line with increases in deltamethrin resistance), normalised mRNA levels were compared between each time point for each population separately using a two-tailed $t$-test $(P<0.05)$.

\section{Supporting data}

The data sets supporting the results of this article are included within the article and as additional files. Microarray data are available in the ArrayExpress database (www.ebi.ac.uk/arrayexpress) under accession numbers E-MTAB-2859 and E-MTAB-2875.

\section{Additional files}

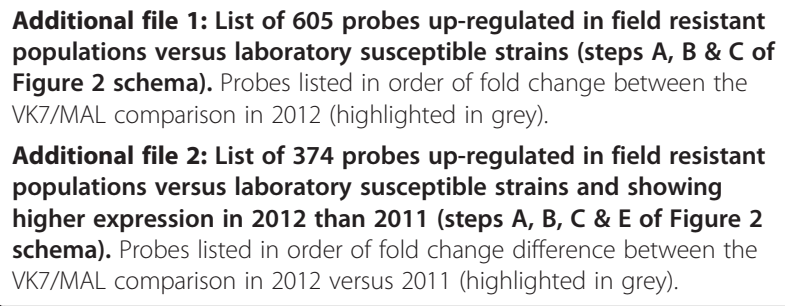

Additional file 2: List of $\mathbf{3 7 4}$ probes up-regulated in field resistant populations versus laboratory susceptible strains and showing higher expression in 2012 than 2011 (steps A, B, C \& E of Figure 2 schema). Probes listed in order of fold change difference between the VK7/MAL comparison in 2012 versus 2011 (highlighted in grey).

Additional file 3: List of 157 candidate probes for deltamethrin resistance in VK7. Probes listed in order of fold change between the VK7/MAL comparison in 2012 (highlighted in grey).

Additional file 4: List of probes showing over-expression in Tengrela compared to VK7 in 2012. Probes listed in order of fold change between TEN versus VK7 in 2012 (highlighted in grey).

Additional file 5: Over-expressed genes from three populations of An. coluzzi from Côte d'Ivoire associated with deltamethrin resistance that are also up-regulated in VK7 in this study. The fold-changes for three populations (Bouaké, M'Be and Tiassalé) compared to N'Gousso are listed with the transcript ID and description.

Additional file 6: List of $\mathbf{2 7 1}$ probes significantly down-regulated in resistant populations versus laboratory susceptible strains and under-expressed in VK7 versus TEN. Probes listed in order of the most under-expressed probes between the VK7/MAL comparison in 2012 (highlighted in grey).

Additional file 7: Information on primers used for $\mathrm{QPCR}$ validation of candidate genes.

\section{Competing interests}

The authors state that they have no competing interests. The funders of the study had no role in the study design or outputs.

\section{Authors' contributions}

KHT and CMJ performed the experiments and analysed the data. HR and CMJ designed the study and KHT, CMJ and HR wrote the manuscript. NS and RKD supervised the field work. All authors read and approved the final version.

\section{Acknowledgements}

The research leading to these results has received funding from the European Union Seventh Framework Programme FP7 (2007-2013) under grant agreement no 265660 AvecNet. We thank Dr. Clare Strode for sharing microarray data from Côte d'Ivoire and Ms Victoria Ingham, LSTM, for assistance with analysis of this data.

\section{Author details}

'Department of Vector Biology, Liverpool School of Tropical Medicine, Liverpool L3 5QA, UK. 'Centre National de Recherche et de la Formation sur le Paludisme, Ouagadougou 01BP 2208, Burkina Faso. ${ }^{3}$ Institut de Recherche en Sciences de la Santé/Centre Muraz, Bobo-Dioulasso 01 BP 545, Burkina Faso.

Received: 18 September 2014 Accepted: 12 February 2015 Published online: 01 March 2015

\section{References}

1. WHO Global Malaria Programme. Global plan for insecticide resistance management in malaria vectors (GPIRM). Geneva: World Health Organisation; 2012

2. Chandre F, Darrier F, Manga L, Akogbeto M, Faye O, Mouchet J, et al. Status of pyrethroid resistance in Anopheles gambiae sensu lato. Bull World Health Organ. 1999:77(3):230-4.

3. Dabiré KR, Diabaté A, Namountougou M, Djogbenou L, Wondji C, Chandre $F$, et al. Trends in insecticide resistance in natural populations of malaria vectors in Burkina Faso, west Africa: 10 Years' surveys. In: Perveen F, editor. Insecticides - pest engineering. Intech. vol 22. 2012. p. 479-502.

4. Diabaté A, Baldet T, Chandre F, Akoobeto M, Guiguemde RT, Darriet F, et al. The role of agricultural use of insecticides in resistance to pyrethroids in Anopheles gambiae s.l. in Burkina Faso. Am J Trop Med Hyg. 2002;67(6):617-22.

5. Toé HK, Jones CM, N'Fale S, Ismail HM, Dabire R, Ranson H. Increased pyrethroid resistance in malaria vectors and reduced bed net effectiveness, Burkina Faso. Emerg Infect Dis. 2014;20(10):6.

6. Hemingway J, Hawkes NJ, McCarroll L, Ranson H. The molecular basis of insecticide resistance in mosquitoes. Insect Biochem Mol Biol. 2004;34(7):653-65. 
7. Donnelly MJ, Corbel V, Weetman D, Wilding CS, Williamson MS, Black WC. Does kdr genotype predict insecticide-resistance phenotype in mosquitoes? Trends Parasitol. 2009;25(5):213-9.

8. Lynd A, Weetman D, Barbosa S, Yawson AE, Mitchell S, Pinto J, et al. Field, genetic, and modeling approaches show strong positive selection acting upon an insecticide resistance mutation in Anopheles gambiae s.s. Mol Biol Evol. 2010;27(5):1117-25.

9. David JP, Strode C, Vontas J, Nikou D, Vaughan A, Pignatelli PM, et al. The Anopheles gambiae detoxification chip: a highly specific microarray to study metabolic-based insecticide resistance in malaria vectors. Proc Natl Acad Sci U S A. 2005;102(11):4080-4.

10. Mitchell SN, Stevenson BJ, Muller P, Wilding CS, Egyir-Yawson A, Field SG, et al. Identification and validation of a gene causing cross-resistance between insecticide classes in Anopheles gambiae from Ghana. Proc Natl Acad Sci U S A. 2012;109(16):6147-52.

11. Jones CM, Toe HK, Sanou A, Namountougou M, Hughes A, Diabate A, et al Additional selection for insecticide resistance in urban malaria vectors: DDT resistance in Anopheles arabiensis from Bobo-Dioulasso. Burkina Faso PLoS One. 2012;7(9):e45995.

12. Muller P, Warr E, Stevenson BJ, Pignatelli PM, Morgan JC, Steven A, et al. Field-caught permethrin-resistant Anopheles gambiae overexpress CYP6P3, a P450 that metabolises pyrethroids. PLoS Genet. 2008;4(11):e1000286.

13. Fossog Tene B, Poupardin R, Costantini C, Awono-Ambene P, Wondji CS,

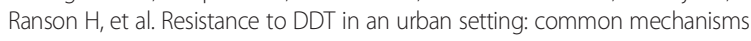
implicated in both $\mathrm{M}$ and $\mathrm{S}$ forms of Anopheles gambiae in the city of Yaounde Cameroon. PLoS One. 2013;8(4):e61408.

14. Stevenson BJ, Bibby J, Pignatelli P, Muangnoicharoen S, O'Neill PM, Lian LY, et al. Cytochrome P450 6M2 from the malaria vector Anopheles gambiae metabolizes pyrethroids: sequential metabolism of deltamethrin revealed. Insect Biochem Mol Biol. 2011;41(7):492-502

15. Edi CV, Djogbenou L, Jenkins AM, Regna K, Muskavitch MA, Poupardin R, et al. CYP6 P450 enzymes and ACE-1 duplication produce extreme and multiple insecticide resistance in the malaria mosquito anopheles gambiae. PLoS Genet. 2014;10(3):e1004236.

16. Chandor-Proust A, Bibby J, Regent-Kloeckner M, Roux J, Guittard-Crilat E, Poupardin R, et al. The central role of mosquito cytochrome P450 CYP6Zs in insecticide detoxification revealed by functional expression and structural modelling. Biochem J. 2013;455:75-85.

17. Jones CM, Haji KA, Khatib BO, Bagi J, Mcha J, Devine GJ, et al. The dynamics of pyrethroid resistance in Anopheles arabiensis from Zanzibar and an assessment of the underlying genetic basis. Parasit Vectors. 2013;6(1):343.

18. Schmittgen TD, Livak KJ. Analyzing real-time PCR data by the comparative C-T method. Nat Protoc. 2008;3(6):1101-8

19. Jones CM, Liyanapathirana M, Agossa FR, Weetman D, Ranson H, Donnelly MJ, et al. Footprints of positive selection associated with a mutation (N1575Y) in the voltage-gated sodium channel of Anopheles gambiae. Proc Natl Acad Sci U S A. 2012;109(17):6614-9.

20. Muller P, Chouaibou M, Pignatelli P, Etang J, Walker ED, Donnelly MJ, et al. Pyrethroid tolerance is associated with elevated expression of antioxidants and agricultural practice in Anopheles arabiensis sampled from an area of cotton fields in Northern Cameroon. Mol Ecol. 2008;17(4):1145-55.

21. Feyereisen R. Insect CYP genes and P450 enzymes. In: Lawrence IG, editor. Insect molecular biology and biochemistry. San Diego: Academic; 2012. p. 236-316.

22. Qiu Y, Tittiger C, Wicker-Thomas C, Le Goff G, Young S, Wajnberg E, et al. An insect-specific P450 oxidative decarbonylase for cuticular hydrocarbon biosynthesis. Proc Natl Acad Sci U S A. 2012;109(37):14858-63.

23. Jasrapuria S, Specht CA, Kramer KJ, Beeman RW, Muthukrishnan S. Gene families of cuticular proteins analogous to peritrophins (CPAPs) in tribolium castaneum have diverse functions. PloS One. 2012;7(11):e49844.

24. Nkya TE, Akhouayri I, Poupardin R, Batengana B, Mosha F, Magesa S, et al. Insecticide resistance mechanisms associated with different environments in the malaria vector Anopheles gambiae: a case study in Tanzania. Malar J. 2014;13:28.

25. Spring JH, Robichaux SR, Hamlin JA. The role of aquaporins in excretion in insects. J Exp Biol. 2009;212(Pt 3):358-62

26. Yang J, McCart C, Woods DJ, Terhzaz S, Greenwood KG, Ffrench-Constant $\mathrm{RH}$, et al. A Drosophila systems approach to xenobiotic metabolism. Physiol Genomics. 2007;30(3):223-31.

27. Munday JC, Eze AA, Baker N, Glover L, Clucas C, Aguinaga Andres D, et al. Trypanosoma brucei aquaglyceroporin 2 is a high-affinity transporter for pentamidine and melaminophenyl arsenic drugs and the main genetic determinant of resistance to these drugs. J Antimicrob Chemother. 2014;69(3):651-63.

28. Giannoni F, Muller HM, Vizioli J, Catteruccia F, Kafatos FC, Crisanti A. Nuclear factors bind to a conserved DNA element that modulates transcription of Anopheles gambiae trypsin genes. J Biol Chem. 2001;276(1):700-7.

29. Gong M, Shen B, Gu Y, Tian H, Ma L, Li X, et al. Serine proteinase overexpression in relation to deltamethrin resistance in Culex pipiens pallens. Arch Biochem Biophys. 2005;438(1):53-62.

30. Kwiatkowska RM, Platt N, Poupardin R, Irving H, Dabire RK, Mitchell S, et al. Dissecting the mechanisms responsible for the multiple insecticide resistance phenotype in Anopheles gambiae s.S. M form, from Vallée du Kou, Burkina Faso. Gene. 2013;519(1):98-106

31. Mitchell SN, Rigden DJ, Dowd AJ, Lu F, Wilding CS, Weetman D, et al. Metabolic and target-site mechanisms combine to confer strong DDT resistance in Anopheles gambiae. PloS One. 2014;9(3):e92662.

32. Nardini L, Christian RN, Coetzer N, Ranson H, Coetzee M, Koekemoer LL. Detoxification enzymes associated with insecticide resistance in laboratory strains of Anopheles arabiensis of different geographic origin. Parasit Vectors. 2012;5:113

33. Edi CVA, Koudou BG, Jones CM, Weetman D, Ranson H. Multiple-insecticide resistance in Anopheles gambiae mosquitoes, Southern Cote d'Ivoire. Emerg Infect Dis. 2012;18(9):1508-11.

34. Ranson H. Facing the resistance crisis in malaria control by developing and evaluating 'Resistance-Breaking' products. Outlooks Pest Manage. 2014;25(1):33-5.

35. Livak K. Organization and mapping of a sequence on the Drosophila melanogaster $X$ and $Y$ chromosomes that is transcribed during spermatogenesis. Genetics. 1984;107(4):611-34.

36. Santolamazza F, Mancini E, Simard F, Qi Y, Tu Z, della Torre A. Insertion polymorphisms of SINE200 retrotransposons within speciation islands of Anopheles gambiae molecular forms. Malar J. 2008;7:163.

37. Bass C, Nikou D, Donnelly MJ, Williamson MS, Ranson H, Ball A, et al. Detection of knockdown resistance $(k d r)$ mutations in Anopheles gambiae: a comparison of two new high-throughput assays with existing methods. Malar J. 2007;6:111

38. Barrett JC, Fry B, Maller J, Daly MJ. Haploview: analysis and visualization of LD and haplotype maps. Bioinformatics. 2005;21:263-5.

39. Coetzee M, Hunt R, Wilkerson R, Torre AD, Coulibaly M, Besansky N. Anopheles coluzzii and Anopheles amharicus, new members of the Anopheles gambiae complex. Zootaxa. 2013;3619(3):246-74.

40. Churchill G. Fundamentals of experimental design for cDNA microarrays. Nat Genet. 2002;32:490-5.

41. Cui $X$, Churchill G. Statistical tests for differential expression in CDNA microarray experiments. Genome Biol. 2003;4(4):210.

42. Smyth G. Limma: Linear models for microarray data. In: Gentleman R, Carey V, Dudoit S, Irizarry R, Huber W, editors. Bioinformatics and computational biology solutions using R and BIOCONDUCTOR. New York: Springer; 2005. p. 397-420.

43. Wu P, Kerr M, Cui X, Churchill G. MAANOVA: a software package for the analysis of spotted cDNA microarray experiments. In: Parmigiani G, Garrett E, Irizarry $\mathrm{R}$, Zeger $\mathrm{S}$, editors. The analysis of gene expression data. Springer: London; 2003. p. 313-41.

\section{Submit your next manuscript to BioMed Central and take full advantage of:}

- Convenient online submission

- Thorough peer review

- No space constraints or color figure charges

- Immediate publication on acceptance

- Inclusion in PubMed, CAS, Scopus and Google Scholar

- Research which is freely available for redistribution 